\title{
Integrando e ampliando a base teórica da criação de valor por empresas geograficamente concentradas
}

\author{
Integrating and expanding the theoretical basis of creating value from firms \\ geographically concentrated
}

\author{
Vinicius Farias Moreira ${ }^{1}$ \\ Walter Fernando Araújo de Moraes ${ }^{2}$ \\ Yákara Vasconcelos Pereira ${ }^{3}$
}

\begin{abstract}
Resumo
Este ensaio teórico visa compreender as fontes de criação de valor por parte das empresas geograficamente concentradas. Seis grupos de recursos do cluster foram identificados, quais sejam: de Produção; de Demanda; de Oferta e Indústrias de Apoio; de Relacionamento no Nível Horizontal; de Relacionamento com as Instituições Locais; e Global Pipelines. Os cinco primeiros representam as múltiplas interações e fluxos de conhecimentos locais entre as empresas e a atmosfera do cluster (local buzz), já o sexto elemento ( $g$ lobal pipelines) representa o reconhecimento dos vínculos externos de relacionamentos, sinalizando que os clusters não são autossuficientes em termos de criação do conhecimento.
\end{abstract}

\begin{abstract}
This theoretical essay aims to understand the creating sources value from firms geographically concentrated. The result of this theoretical articulation was the identification of six cluster resource groups: Production; Demand; Offer and Industry Support; Relationship in the Horizontal Level; Relationship with local institutions; and Global Pipelines. The first five represent the multiple interactions and flows of local knowledge between firms and the atmosphere of the cluster (local buzz) and the sixth element (global pipelines) represents the recognition of external relationships bonds, signaling that the clusters are not self-sufficient in terms of knowledge creation.
\end{abstract}

\section{Preliminares acerca da concentração geográfica de empresas}

Este estudo reconhece que as bases de criação de valor das empresas podem ir além das contribuições da visão baseada nos recursos (BARNEY, 1991; DIERICKX; COOL, 1989; GRANT, 1991; PETERAF, 1993) e da abordagem das capacidades dinâmicas (EISENHARDT; MARTIN, 2000; PETERAF; STEFANO; VERONA, 2013; TEECE; PISANO; SHUEN, 1997). Ao se buscar compreender as empresas geograficamente concentradas, assume-se o entendimento de que as externalidades da localidade podem representar importantes fatores competitivos (HERVAS-OLIVER; ALBORS-GARRIGÓS, 2007; 2009; MARTINEZ-FERNANDEZ, 2004; MCEVILY; ZAHEER, 1999; MOLINA-MORALES), que, entre outros aspectos, consideram as contribuições ligadas à visão relacional (DYER; SIGHT, 1998; GIULIANI, 2013) e às instituições (MALMBERG; MASKELL, 2002; NORTH, 1990; PENG et al., 2008).

Historicamente, a constatação de que a concentração geográfica pode ser fator de sucesso no desempenho é remetida às contribuições de Alfred Marshall ao observar as configurações industriais na Inglaterra do século XIX e retratar o fenômeno do industrial district, evidenciando que, em uma aglomeração

Mestre e doutor em Administração pelo Programa de Pós-graduação em Administração da Universidade Federal de Pernambuco (PROPAD/ UFPE). Afiliação: UFCG. Brasil. Lattes: http://lattes.cnpq.br/3481559213937969 Email: viniciusfmoreira@yahoo.com.br

2 Doutorado em Management Sciences - University of Manchester Institute of Science and Techonology. Afiliação: UFPE. Brasil. Lattes: http:// lattes.cnpq.br/6093921820124337 Email: wfam1950@gmail.com

3 Doutorado (2012- bolsista CAPES), mestrado (2006- bolsista) e graduação (2002) em Administração (UFPE). Afiliação: UFPB. Brasil. Lattes: http://lattes.cnpq.br/3420201580418099 Email: yakarav@gmail.com 
territorial de empresas de um mesmo ramo ou de ramo similar, externalidades positivas são geradas, sendo representadas pela tríade: mão de obra especializada, spillovers tecnológicos, e vínculos de oferta e demanda de bens intermediários. Observando essa realidade, Marshall (1920) percebeu a existência de uma atmosfera industrial favorável, em que a acumulação e concentração local de conhecimentos, habilidades e knowhow foram capazes de gerar benefícios econômicos e difundir a inovação.

Esses conhecimentos foram resgatados, no final dos anos 70 e início dos anos 80, com uma revisita ao modelo original de Marshall por Brusco (1982) e Becattini (1990), que fazem um esforço para explicar anomalias socioeconômicas da terceira Itália. Esses estudos remetem a análises de indústrias italianas tradicionais e concentradas, fazendo associações entre os modelos de industrialização e o desenvolvimento econômico (SFORZI, 2008). Dessas contribuições iniciais, surgem as bases para o entendimento do que vem a ser aglomerações, tratando-se do fenômeno em que empresas dentro de uma mesma indústria ou indústrias relacionadas tendem a se reunir em uma mesma região, proporcionando o que se convenciona por economias da localização (MALMBERG; MASKELL, 2002). Ao longo dos anos 90, além das experiências italianas, registra-se a experiência americana, especificamente a denominada "escola californiana" de aglomerações de alta tecnologia, com destaque para o papel das instituições e dos elos de interdependência, e para as experiências da indústria química alemã e da eletrônica japonesa (LASTRESet al., 2006; PORTER, 1991).

A relevância do fenômeno tem impulsionado estudos que analisam as vantagens da localidade sobre diferentes lentes teóricas, incluindo administração, economia, sociologia, geografia econômica, estudos regionais, planejamento urbano, inovação, entre outros (HERVAS-OLIVER; ALBORS-GARRIGÓS, 2009; LAZZERETTI et al., 2013; ZEN, 2010). De uma forma ou de outra, o nível microeconômico da firma no cluster tem recebido menor atenção pelo mainstream entre os estudos de cluster (GIULIANI, 2007; 2013;HERVAS et al., 2012;MOLINA-MORALES; MARTINEZ-FERNANDEZ, 2004; RIGBY; BROWN, 2013; TER WAL; BOSCHMA, 2011), e isso reforça a motivação em responder à seguinte questão de pesquisa: quais as fontes de criação de valor para empresas em cluster?

O surgimento de aglomerações territoriais, clusters ou distritos industriais geralmente está associado à preexistência de determinados aspectos, tais como: mão de obra especializada, recursos naturais, entradas de empresas com dinâmicas externas (multinacionais ou subsidiárias) e a presença de instituições locais ou empresas âncoras que levem à propagação de novos negócios no território, o que acontece por meio dos spillovers de conhecimento (MALMBERG; MASKELL, 2010). Uma vez que o cluster começa a se formar, o ciclo de auto reforço promove seu crescimento, especialmente quando as instituições locais asseguram suporte (PORTER, 1998).

Os termos utilizados para análise das vantagens da localidade são os mais diversos, incluindo: distritos industriais; cluster industrial; aglomerações industriais; arranjos produtivos e inovativos locais; sistemas de produção localizado; distritos tecnológicos; entre outros (GIULIANI; BELL, 2005; LASTRESet al., 2006; MALMBERG; MASKELL, 2002; PORTER, 1998). Mesmo assim, é importante reconhecer que tais aglomerações foram popularizadas na literatura específica como clusters, destacando sua importância, no contexto nacional e internacional, pela geração de empregos, crescimento econômico, desenvolvimento tecnológico e potencial exportador (SUZIGAN; GARCIA; FURTADO, 2005).

Neste estudo, assume-se como referência o conceito de cluster estabelecido por Michael Porter, autor que o introduziu para a Administração Estratégica (LAZZERETTI et al., 2013), sendo um grande entusiasta para acadêmicos e desenvolvedores de políticas públicas (MCCANN; FOLTA, 2008), além de ser a referência mais citada pelos trabalhos acadêmicos relacionados à temática (ARTUSO et al., 2012). Entende-se cluster como "[...] um grupo geograficamente próximo de empresas interligadas e instituições associadas em um campo particular, ligadas por interesses comuns e complementares" (PORTER, 2000, p.16). Ao buscar identificar a vantagem competitiva dos países, Porter (1991) evidenciou a existência de clusters como fenômenos propulsores de desempenho superior, a despeito do desempenho dos países.

Trata-se aqui de um ensaio teórico acerca de cluster, tendo como objetivo compreender as fontes de criação de valor por parte das empresas geograficamente concentradas. Além da respectiva empresa ser pouco estudada pelo mainstream da área, a identificação dos elementos que compõem a atmosfera do cluster ou local buzz (BATHELTet al., 2004) é muitas vezes confusa e dispersa na literatura (LAZZERETTI 
et al., 2013). O reconhecimento dessas fontes de criação de valor representa um caminho potencial para as empresas e clusters alcançarem e/ou sustentarem suas vantagens competitivas.

\section{Contribuições teóricas para análise da concentração geográfica de empresas}

Aqui há um esforço em destacar contribuições teóricas advindas de uma revisão na literatura especializada em Administração Estratégica, com destaque para as contribuições dos grupos de atributos do modelo Diamante (PORTER, 1991; 1998; 2000); e aos esforços em trazer as contribuições da visão baseada nos recursos para a análise territorial (FOSS, 1996;HERVÁS-OLIVER; ALBORS-GARRIGOS, 2007; 2009; MOLINA-MORALES; MARTINEZ-FERNANDEZ, 2004), o que é lastreado pelos conhecimentos da visão baseada no conhecimento (MALMBERG; MASKELL, 2002; TALLMAN et al., 2004). Os discursos de cada uma dessas perspectivas não se anulam e podem ser vistos de forma complementar para um entendimento mais amplo das vantagens da localidade.

Tomando por base as críticas às suas contribuições iniciais (PORTER, 1979, 1985), e fazendo parte mesmo da sua evolução do pensamento, Porter (1991) faz uma revisão do progresso do campo da estratégia em direção ao desenvolvimento de uma teoria dinâmica da estratégia. Para tanto, o autor passa a considerar as escolhas gerenciais, oriundas da análise do ambiente externo e interno, associadas às condições iniciais da firma ou do ambiente em que ela está situada. Além disso, reconhece a contribuição dos modelos de teoria dos jogos, por perceber que o padrão das escolhas estratégicas dos concorrentes também é dinâmico; a influência dos compromissos e incertezas do ambiente; e as contribuições da teoria baseada nos recursos.

Essa construção teórica faz com que Porter (1991) deduza que o sucesso pode estar geograficamente localizado, o que ele chama de vantagem competitiva das nações, servindo de base para explicar clusters de indústrias competitivas. Essa estrutura é captada pelo modelo Diamante, composto por quatro amplos atributos do ambiente próximo à firma e que influenciam na capacidade de inovar e estabelecer um upgrade, são eles: condições dos fatores; condições de demanda; indústrias correlatas e de apoio; e estratégia, estrutura e rivalidade da firma. Esses atributos estabelecem entre si relações de interdependência e reforço mútuo. Ao passo em que são analisados, apontam o caminho pelo qual as firmas têm de perceber oportunidades e estarem atentas às pressões que sofrem para agir e manterem-se competitivas. Além disso, o modelo prevê, ainda, que o governo e o acaso podem explicar parte do sucesso competitivo das empresas.

Por sua vez, as contribuições advindas da perspectiva dos recursos internos têm sido inspiradoras aos pesquisadores organizacionais que buscam compreender a influência do território no desempenho das empresas (HERVAS-OLIVER; ALBORS-GARRIGÓS, 2007; 2009; MOLINA-MORALES; MARTINEZFERNANDEZ, 2004). Essa análise é especialmente necessária quando se deseja entender a dinâmica da criação de valor de empresas geograficamente concentradas, em que os recursos estratégicos não podem ser entendidos sem que seja considerada a influência dos recursos compartilhados ou dos recursos da localidade. O argumento é o de que os territórios contêm capacidades de alta ordem (FOSS, 1996) disponíveis para as firmas locais e que contribuem para explicar a dinâmica dos recursos internos (BATHELT et al., 2004). As interações entre os recursos internos das empresas e os recursos da localidade possibilitam que as empresas? sejam heterogêneas por natureza, dado os aspectos contextuais em que os recursos e capacidades se combinam, além de outras interações dinâmicas que estabelecem no ambiente em que operam (HERVAS-OLIVER; ALBORS-GARRIGÓS, 2007).

Os recursos da localidade representam aqueles recursos e capacidades intangíveis compartilhados pelas empresas de um mesmo distrito industrial (cluster), e sugerem a aplicação da mesma lógica direcionada ao alcance da vantagem competitiva sustentável (BARNEY, 1991), sendo de difícil compreensão às empresas que estão externas ao cluster. Esse tipo de conhecimento é baseado nas rotinas ligadas à história das empresas, às práticas de negócios, às instituições específicas e aos múltiplos links entre os atores (MOLINA-MORALES; MARTINEZ-FERNANDEZ, 2004). Essa perspectiva é fortemente influenciada pela Teoria Baseada no Conhecimento, cuja ênfase é para as interações e trocas de conhecimentos entre as empresas do clustere as capacidades de criação de conhecimento do cluster (ARIKAN, 2009; MALMBERG; MASKELL, 2002; TALLMAN et al., 2004), o que representa uma compreensão adicional à sustentabilidade da 
vantagem competitiva para clusters e as empresas dentro deles (HERVAS-OLIVER; ALBORS-GARRIGÓS, 2007; TALLMAN et al., 2004).

Para compreender essa lógica, admite-se a existência de estoques de conhecimentos específicos da empresa e do cluster, entendendo que a especificidade de cada nível está protegida, em partes, pela assimetria nos fluxos de conhecimento. Essas assimetrias resultam da dinâmica de interação do conhecimento dentro da empresa, e entre a empresa e a dinâmica do cluster que a envolve (ARIKAN, 2009; TALLMAN et al., 2004). Tallman et al.(2004) evidenciam duas modalidades de conhecimentos: os específicos das empresas e os específicos do cluster. Em cada uma dessas modalidades é possível acessar o tipo de conhecimento componente (elementos identificáveis no corpo de conhecimento, relativamente transparente, podendo ir do técnico ao sistemático, e relativamente de fácil mobilidade entre as empresas) e o conhecimento arquitetônico (um sistema complexo de rotinas organizacionais que coordena e integra os conhecimentos componentes, seja da empresa, seja do cluster, e tem natureza enraizada, não transparente e com causas ambíguas, além de ser relativamente imóvel entre organizações). Analisando pela perspectiva das empresas, esses conteúdos perpassam pelos conceitos de rotinas (NELSON; WINTER, 1982), recursos organizacionais (BARNEY, 1991) e capacidades dinâmicas (EISENHARDT; MARTIN, 2000; TEECE; PISANO; SHUEN, 1997).

A assimilação dos conhecimentos do cluster pelas empresas membros vai depender da capacidade absortiva de cada empresa e de seus recursos e capacidades que moderam a combinação e o processo de acumulação (HERVAS-OLIVER; ALBORS-GARRIGÓS, 2007). Mesmo considerando as diferenças particulares de conhecimento de cada firma, a capacidade absortiva delas dentro do cluster tende a ser próxima, tendo em vista a força das múltiplas e dinâmicas interdependências que acontecem dentro dele (TALLMAN et al., 2004). Por sua vez, ao comparar com empresas fora do cluster, as diferenças tendem a se revelar mais aparentemente enraizadas em um componente de conhecimento sistemático e tácito, que requer um profundo entendimento de seus significados, muito mais que um simples know-how técnico, indo além do que se convenciona como compensações de mercado e não sendo formalmente transmitido, representando parte de um conhecimento comum aos membros, cujo controle do fluxo de informações é virtualmente impossível (TALLMAN et al., 2004).

As explicações para essa atmosfera coletiva podem passar pelo que se convenciona por embeddedness tecnológicos. O conceito de embeddedness, embora seja atribuído a Karl Polanyi, foi difundido por Granoveter (1985), para quem representa o processo em que interesses econômicos almejados pelas empresas caminham em paralelo com o contexto social no qual elas estão inseridas, de maneira que a análise isolada de instituições poderá acarretar em um grande equívoco. Assim, considera-se que a economia está imersa nas relações sociais, não se mostrando desvinculada da sociedade (GRANOVETER, 1985; NELSON, 2002). O conceito subsidia, especialmente, o entendimento dos elos de relacionamento entre as empresas (MCEVILY; ZAHEER, 1999), mas também demonstra oportunidades múltiplas dentro do cluster, entre as quais está o desenvolvimento de novos negócios, ao considerar lacunas de produtos e serviços dentro do aglomerado, a existência de conhecimento específico dentro da localidade, de instituições financeiras e investidores, além da presença de um mercado local e relacionamentos (PORTER, 1998).

Entende-se que um cluster é geralmente definido tomando por base a dimensão horizontal em que muitas empresas operam em uma mesma indústria, ou seja, produzem um produto final similar (MALMBERG; MASKELL, 2002). Dentro dessa atmosfera do cluster as empresas interligadas teriam vantagens adicionais como o fácil acesso a fornecedores especializados de matéria prima e equipamentos, canais facilitados para clientes, provedores de serviços, empresas de indústrias relacionadas e instituições associadas (universidades, agências de apoio e associações comerciais), ao tempo em que competem e também cooperam entre si (GIULIANI, 2013; MOLINA-MORALES; MARTINEZ-FERNANDEZ, 2004; PORTER, 1998; 2000). Nessa atmosfera (ou local buzz), as interações e trocas de informações são frequentes, muitas vezes de forma não estruturada e automática, dentro de uma mesma indústria, lugar ou região, permitindo esquemas interpretativos e entendimentos semelhantes, estimulado pelo estabelecimento de convenções e outros arranjos institucionais (BATHELT et al., 2004).

A integração entre as abordagens apresentadas permite uma sistematização das fontes de criação de valor por parte das empresas geograficamente concentradas, aqui expressa por seis grupos de recursos: 
de Produção, de Demanda, de Oferta e Indústrias de Apoio, de Relacionamento no Nível Horizontal, Relacionamento com Instituições Locais e Global Pipelines.

\subsection{Recursos de Produção}

Na estrutura do cluster, os recursos de produção são altamente especializados, podendo ser herdados ou criados por meio de investimentos privados e sociais. O spillover de conhecimento gerado pelo ambiente possibilita o desenvolvimento em quantidade e com menor custo dos recursos naturais, humanos e de capital, além de viabilizar uma infraestrutura física, administrativa, de informação, científica e tecnológica, que favorecem fatores especializados e de qualidade (MALMBERG; MASKELL, 2002; PORTER, 2000). Entre os fatores imóveis de produção, Nelson (1996) destaca o clima, a fertilidade do solo e os recursos naturais. Os recursos naturais poderão, inclusive, atuar como impulsionadores do surgimento de clusters (MARSHALL, 1920), representando uma aproximação das empresas às fontes de matéria-prima (RIGBY; BROWN, 2013). A existência desses recursos e condições de infraestrutura seguirá em maior ou menor intensidade em função do aspecto temporal, o que faz revelar a perspectiva dinâmica desses fatores.

Acerca dos recursos humanos, essas regiões geográficas desencadeiam atratividade de operários especializados oriundos de regiões distantes e que visualizam no cluster boas perspectivas profissionais, ao passo que educa os moradores locais. As empresas inseridas em clusters vibrantes poderão ter acesso a um conjunto de profissionais especializados e experientes, e isso poderá representar redução do tempo e nos recursos financeiros dispendidos com processos de recrutamento (ASHEIM; GERTLER, 2005; KRUGMAN, 1991;MARSHALL, 1920; NELSON, 1996; PORTER, 1998). Uma ressalva a esse argumento é apresentada pelo estudo de McCann e Folta (2008), que indicam que fases avançadas no ciclo de vida do cluster podem implicar em maiores custos com mão de obra especializada (valor da hora de trabalho), o que poderia representar uma externalidade negativa para algumas empresas.

\subsection{Recursos de demanda}

Do ponto de vista dos recursos de demanda, a condição das empresas estarem geograficamente concentradas gera uma série de ganhos em termos de complementariedades, entre esses: o fato de que os consumidores tendem a perceber de forma mais valiosa os produtos da região face à boa reputação que a localidade possa gerar; a possibilidade de alcançar múltiplos fornecedores, o que impulsiona a demanda de clientes em relação ao cluster, e o fato de que os consumidores mais sofisticados tendem a alcançarem melhor satisfação de suas necessidades nos clusters do que nas empresas que competem isoladamente (PORTER, 1998).

O entendimento dos fatores de demanda auxiliam as empresas do aglomerado a partir do nível de sofisticação e especialização da demanda local, que impulsiona as empresas a saírem da competição baseada na baixa qualidade e baixo custo, para uma competição baseada na diferenciação. Essa situação pressiona as empresas a melhorarem e perceberem as necessidades existentes e futuras que são difíceis de serem constatadas nos mercados internacionais, além disso, podem revelar segmentos de mercado onde as empresas podem se diferenciar (PORTER, 2000).

Tratando-se de clusters com índices de internacionalização, vale o entendimento das Cadeias de Valor Globais (Global Value Chain), com destaque ao papel dos compradores globais (Global Buyers) na formação e coordenação de uma produção fragmentada, além do entendimento das lógicas associadas às redes de distribuição ao longo da cadeia de valor. Um dos desafios associados é a coordenação dos fornecedores quanto às exigências requeridas em cada tipo de mercado, isso porque geralmente há diferenças entre as exigências determinadas pelo mercado doméstico e as internacionais, apontando para a necessidade de um alto grau de monitoramento e controle (GEREFFI et al., 2005).

Analisando os compradores globais no setor agrícola, Humphrey e Memedovic (2006) destacam que esses têm assumido uma importância cada vez maior em determinar parte dos processos produtivos das empresas produtoras, o que inclui a oferta de grandes volumes, velocidade e confiabilidade da entrega, customização do produto por meio do processamento, embalagem e garantia sobre a qualidade do produto. 
Nesse setor, os compradores globais podem estar representados pelas grandes redes de supermercados ou importadoras a elas associadas, que tendem a estabelecer elos complexos e muitas vezes relacionais de interações, mantendo o compromisso de responder a um ambiente regulatório intricado, às questões de segurança alimentar (particularmente resíduos de pesticidas e condições de processamento pós-colheita), assim como às normas ambientais e trabalhistas (GEREFFI et al., 2005).

\subsection{Recursos de oferta e indústrias de apoio}

Estando inseridas em clusters é provável que as empresas percebam a presença de uma base de fornecedores especializados e sólida, o que poderá representar baixo custo de transação para suas operações. Essa situação tende a minimizar os custos de importação e a possibilidade de atrasos nas entregas, além de reduzir o risco dos fornecedores superfaturar ou renegar seus compromissos. Sobre os compromissos, a atmosfera do cluster propicia o desenvolvimento de alianças informais com os fornecedores, o que de certa forma favorece a flexibilidade organizacional. Considerando o provável volume de vendas, os fornecedores buscam acompanhar de perto as necessidades das empresas, apoiando no desenvolvimento dos produtos e assegurando maquinários e serviços especializados para o processo de inovação na indústria, o que busca garantir maior eficiência e velocidade de mercado (EISINGERICH et al., 2010; PORTER, 1991).

Muitas vezes essa cadeia de apoio é responsável pela disseminação da inovação entre as empresas do cluster. Hervas-Oliver e Albors-Garrigós (2007) ilustram o cenário em que o conhecimento promovido a partir da interação entre a base de conhecimento da empresa e os recursos coletivos do cluster possibilita o desenvolvimento da inovação, inicialmente protegida para gerar vantagem competitiva para a empresa, mas que ao passar do tempo, esse conhecimento vai ficando parcialmente entendido pelos membros de apoio que podem passar a reproduzi-lo como práticas de benchmarking, incorporando dentro do conhecimento arquitetônico do cluster. Esse conhecimento que gerou vantagem competitiva temporária para uma ou outra empresa, poderá estar gerando vantagem sustentável para as empresas desse determinado cluster face às empresas externas.

As práticas de benchmarking comuns às empresas do cluster na dimensão horizontal podem estar associadas aos processos de aprendizagem localizada, em que efeitos de vizinhança decorrente das diversas interações e encontros entre as empresas levam trocas de informações e benefícios de spillovers de conhecimentos que conduzem as empresas de uma mesma localidade a processos similares de captação e atualização do conhecimento. As explicações estariam no fato de que a proximidade espacial estimula as empresas a desenvolverem linguagem, atitudes tecnológicas e esquemas interpretativos similares (MALMBERG; MASKELL, 2006). A partir desse cenário, essas empresas podem monitorar as práticas das demais sem maiores custos ou esforços, ou seja, as soluções para seus problemas tendem a ser facilmente identificadas e imitadas por meio da combinação com sua base de recursos (MALMBERG; MASKELL, 2002), que, embora similares, nunca serão idênticas (TALLMAN et al., 2004). Essas questões demonstram a convivência entre as situações de competição e cooperação entre as empresas geograficamente agrupadas.

\subsection{Recursos de relacionamento no nível horizontal}

A rivalidade local é um ponto que faz com que as empresas possam se estruturar e se fortalecer frente aos rivais estrangeiros, e sua dinâmica alimenta todos os outros aspectos da estrutura do cluster. Admitese, entretanto, que nos casos em que as empresas dentro do cluster assumir entre si uma competição vigorosa, a lógica da forma de organização espacial poderá falhar (MCCANN; FOLTA, 2008; PORTER, 1998). Já a cooperação, fundamenta-se no fato de que a empresa individualmente teria que vencer grandes obstáculos quando comparados à ação conjunta para ter acesso às oportunidades. As empresas em um cluster usam diversos tipos de networks para acessar o conhecimento de atores locais e dos mais distantes (GIULIANI, 2013). Entre os atores locais, destacam-se os recursos de relacionamento no nível horizontal, tidos como aqueles que foram desenvolvidos e compartilhados pelas empresas produtoras de mercadorias similares e concorrentes entre si no ambiente do cluster,e que geralmente exercem uma influência positiva na competitividade internacional destas (FENSTERSEIFER; WILK, 2005; GIULIANI, 2013; PORTER, 1991; 1998; TER WAL, 2013; ZEN, 2010). 
A visão relacional é explorada por Dyer e Singh (1998), que indicam que a cooperação entre empresas visa estabelecer links de criação de valor entre elas, podendo representar recursos críticos na busca pela vantagem competitiva em ambientes em que as empresas mantêm múltiplos e frequentes relacionamentos colaborativos, sendo capaz de preencher uma lacuna presente tanto na visão dos recursos internos (BARNEY, 1991; GRANT, 1991; PENROSE, 1995) quanto na visão do posicionamento (PORTER, 1981; 1985; 1991). Esses recursos são protegidos por mecanismos que preservam os retornos prometidos (DYER; SIGHT, 1998), o que segue lógica compatível com a proteção dos recursos internos no alcance da vantagem competitiva (BARNEY, 1991; DIERICKX; COOL, 1989), guardados suas devidas proporções analíticas.

A competição coexistirá com a cooperação porque elas ocorrem em diferentes dimensões e entre jogadores diferentes, gerando um ganho mútuo. Os membros das empresas do cluster possuem acesso privilegiado às informações especializadas acerca do mercado, de questões técnicas e ao acúmulo de informações competitivas dentro do aglomerado, afora os ganhos de relacionamentos pessoais e elos comunitários que inspiram confiança entre os diversos agentes que facilitam o fluxo de informação. Por tais razões, os players locais facilmente compartilham custos com laboratórios de testes e centros de qualidade, conhecimentos acerca do custo de insumos e desempenho dos empregados operacionais e especializados, bem como conhecimentos acerca da ação das instituições financeiras (PORTER, 1998). Essas interações são caracterizadas como sendo não estruturadas e não planejadas, relativamente amplas, difusas e frequentemente envolvendo o uso imediato dos conhecimentos (MALMBERG; MASKELL, 2010). Como indicado, esses relacionamentos horizontais são geradores de trocas de conhecimentos valiosas e possibilitam aprendizado interativo entre as empresas envolvidas (HERVAS-OLIVER; ALBORS-GARRIGÓS, 2009; MCEVILY; ZAHEER, 1999). Segundo Tallmanet al. (2004), quanto mais as empresas compartilharem as condições e experiências, maior será a mútua capacidade absortiva em torno dos conhecimentos do cluster.

O fato das empresas estarem geograficamente próximas tende a aumentar não apenas a frequência das interações dentro do cluster, mas também a efetividade das trocas de conhecimento por meio das interações do contato face-a-face entre elas, o que alimenta os elos de confiança e institucionalizam normas de cooperação (ARIKAN, 2009; BATHELT et al., 2004). Trata-se de um ambiente em que gestores e trabalhadores são capazes de discutir os problemas específicos do contexto, vencendo ambiguidades e incertezas por meio da observação direta e confronto, facilitando o desenvolvimento de uma expertise valiosa, que pode ser traduzida em estratégias de diferenciação dos produtos e inovação (GIULIANI, 2013).

O oportunismo entre os atores da rede é supostamente controlado por meio do efeito de repetidas transações, reputação e normas sociais que possam estar vinculadas à localização geográfica em particular ou ao grupo social (GEREFFI et al., 2005). É possível também que, com o passar do tempo, esses relacionamentos se desgastem, e o conhecimento oriundos dos elos não compensem a continuidade dos custos de manutenção, é quando as empresas seguem em busca de novos horizontes para se reoxigenar e continuar crescendo (ARIKAN, 2009). Por fim, embora as redes de relacionamentos sejam promissoras aos seus participantes, seus ganhos de conhecimentos decorrem da capacidade de absorção de cada empresa, o que envolve a habilidade de descobrir e explorar o conhecimento disponível (KOGUT, 2000;MCEVILY; ZAHEER, 1999; TALLMAN et al., 2004).

\subsection{Recursos de relacionamento com as instituições locais}

Ainda analisando os fluxos de conhecimentos em clusters, é imprescindível o entendimento dos recursos de relacionamento com as instituições locais. Estas estão diretamente relacionadas com a promoção do crescimento econômico por meio de uma progressiva introdução de novas tecnologias associadas com o aumento no nível de produtividade dos trabalhadores e com a habilidade de produzir ou melhorar mercadorias e serviços (MCEVILY; ZAHEER, 1999; NELSON; SAMPAT, 2001). As Instituições podem ser públicas ou privadas e buscam promover suporte coletivo para as empresas em um dado distrito ou região, na medida em que fortalece sua competitividade face às empresas de outras regiões (MOLINA-MORALES; MARTINEZFERNANDEZ, 2004), sendo sua análise ainda mais necessária quando se busca entender a competitividade das empresas, nacionais ou estrangeiras, localizadas em países em desenvolvimento (PENG et al., 2008). 
Segundo North (1990), as instituições podem interagir com as empresas de três formas: no papel de regulação (pela adoção de regras e leis específicas), no papel normativo (relativo aos códigos de conduta) e influenciando a o desenvolvimento de uma cultura local específica (o que acontece por meio da construção da identidade dos agentes, modelos mentais, crenças e esquemas interpretativos). Interessa para esse estudo especialmente a condição de suporte empresarial exercida pelas instituições locais.

Esses tipos de interações muitas vezes recebem pouco destaque quando da análise da competitividade das empresas em países desenvolvidos, a exemplo das contribuições trazidas pela proposição analítica do Modelo Diamante (PORTER, 1991). Isso pode ter explicação no contexto para o qual o modelo foi desenvolvido, o mercado americano, que apresenta um conjunto de instituições com comportamentos relativamente estáveis, com orientação focada ao mercado (PENG et al., 2008). Essa explicação certamente atende ao fato de que teorias consagradas para explicar a competitividade empresarial como a perspectiva da indústria (PORTER, 1985) e a perspectiva dos recursos internos (BARNEY, 1991; GRANT, 1991;PENROSE, 1995; PETERAF, 1993; WERNERFELT, 1984) negligenciaram o papel das instituições no que tange às definições de estratégias empresariais.

Aqui, entende-se que as instituições estão muito vinculadas às trajetórias de desenvolvimento do cluster, desenvolvendo encaixes (fits) únicos, os quais também contemplam conexões independentes e informais estabelecidas entre instituições e empresas, que representam uma robusta forma organizacional em busca de vantagens de eficiência, efetividade e flexibilidade (MALMBERG; MASKELL, 2002). Em algumas situações, essas instituições podem assumir as regras do jogo ao coordenar o sistema global de adição de valor às atividades de empresas multinacionais, assumindo papéis essenciais em auxiliar essas empresas na busca por eficiência quanto à aquisição de insumos, produção, marketing e recursos humanos, estabelecendo conexões entre o nível nacional e o da empresa (DUNNING; LUNDAN, 2008).

\subsection{Global Pipelines}

Por último, reforçando e complementando a dinâmica de criação de valor por parte das empresas geograficamente concentradas, é preciso reconhecer os vínculos externos de relacionamentos (HERVASOLIVER et al., 2008; HERVAS-OLIVER; BOIX-DOMENECH, 2012) ou globalpipelines (BATHELT et al., 2004). Essa perspectiva é geralmente negligenciada pelo mainstream, cuja análise tende a centrar-se na perspectiva endógena do cluster, como se o conjunto de indústrias e serviços relacionados fosse autossuficiente (HERVAS-OLIVER et al., 2008). Esses elos são importantes para aumentar a variedade das fontes de conhecimento em um contexto local e evitar que a formação do cluster se torne uma armadilha tecnológica (GIULIANI, 2013). Para Batheltet al. (2004), nenhum cluster pode ser considerado autossuficiente em termos de estado da arte de novos conhecimentos, sendo fundamental o desenvolvimento de pipelines para a busca de fontes de excelências globais.

A inovação, a criação do conhecimento e a aprendizagem decorre do processo interativo em que atores que carregam consigo diferentes bases de conhecimento e competências passam a caminhar juntos e trocar informações com o objetivo de resolver problemas técnicos, organizacionais, comerciais ou intelectuais. Essas trocas envolvem tanto as interações e aprendizados dentro do cluster (local buzz) como as conexões com os agentes externos (global pipelines), fazendo com que as empresas estejam conectadas a uma rede dinâmica de competitividade global (BATHELT et al.,2004).

As conexões externas são geralmente estabelecidas a partir da participação de empresas multinacionais nas Cadeias de Valor Global (GEREFFI et al., 2005; HUMPHREY; MEMEDOVIC, 2006), fazendo as conexões de conhecimento entre clusters (HERVAS-OLIVER; BOIX-DOMENECH, 2012; RIGBY; BROWN, 2013). As subsidiárias das multinacionais fazem parte de um determinado contexto local, tendo, portanto, acesso aos recursos dessa localidade e podendo difundi-los em suas relações em outros clusters no qual a multinacional atua. Dessa forma, as relações estabelecidas com agentes fora do cluster ou com empresas estrangeiras que se localizam em várias regiões geográficas ao mesmo tempo contribuem para a transferência do conhecimento e para a redução das assimetrias de conhecimento territorial (HERVAS-OLIVER et al., 2008). A presença de multinacionais nos clusters regionais representa a possibilidade do estabelecimento de conexões localglobal, sendo um ingrediente fundamental no processo cumulativo de desenvolvimento da empresa e do 
cluster, ao passo que apresenta um impacto positivo e significante nos links de criação do conhecimento e nas redes de relacionamentos extensivas com as empresas locais, o que acaba envolvendo a geração de ativos complementares (IAMMARINO; MCCANN, 2010).Cabe destacar que a assimilação do conhecimento dependerá da capacidade absortiva do cluster e dos próprios recursos e capacidades empresariais que moderam o processo de acumulação (HERVAS-OLIVER; ALBORS-GARRIGÓS, 2007; HERVAS-OLIVER et al., 2008; MCCANN; FOLTA, 2008).

Os global pipelines abrangem empresas de diferentes partes do mundo que estão embebidas em diferentes contextos socioinstitucionais e culturais, e o estabelecimento desses vínculos requer tempo e esforços conscientes e substanciais no sentido de evitar falhas de informações. Além disso, os global pipelinesnão são automáticos nem gratuitos, sendo fruto de investimentos específicos, que ocorrem a partir de um processo complexo e oneroso, requerendo suporte institucional e de infraestrutura, e estando dependentes da confiança estabelecida entre as partes (BATHELT et al.,2004; MOLINA-MORALES; MARTINEZ-FERNANDEZ, 2004).

O desenvolvimento de redes de relacionamentos que funcionem bem como fontes de conhecimento, sejam a partir das interações internas ou por meio das externas, representam uma das mais importantes capacidades localizadas. Por último, vale destacar que o conteúdo, o contexto e o meio aplicado pode prejudicar ou favorecer a formação de redes de colaboração e afetar os caminhos de como elas podem se desdobrar no espaço geográfico (MALMBERG; MASKELL, 2006). Depois de apresentadas as fontes de criação de valor para empresas geograficamente concentradas, seguem as conclusões.

\section{Considerações Finais}

Geralmente a criação de valor estratégico por parte das empresas é explicado por meio da Visão Baseada nos Recursos, esse estudo busca ampliar esse entendimento ao focar análise nas empresas geograficamente concentradas, indicando que outros aspectos relacionados à concentração devem ser considerados como fontes de valor.

Assumiu-se o entendimento de que o desenvolvimento do cluster e o das empresas estão diretamente relacionados, acontecendo de forma concomitante, não sendo suficiente a análise isolada de qualquer desses níveis para a compreensão do fenômeno buscado (HERVAS-OLIVER; BOIX-DOMENECH, 2012;TALLMAN; CHACAR, 2011; TER WAL, 2013). Assim, convém entender que os recursos internos das empresas ajudam a explicar a dinâmica dos conhecimentos do cluster e os recursos do cluster disponíveis para as firmas locais contribuem para explicar a dinâmica dos recursos internos (BATHELT et al., 2004; HERVAS-OLIVER; ALBORS-GARRIGÓS, 2007; 2009;MASKELL; MALMBERG, 1999;MCCANN; FOLTA, 2008).

Além disso, entende-se que os recursos externos da localidade são acessados por meio dos recursos internos das empresas, evidenciando processos de aprendizagem contínuos por meio da combinação e acumulação de conhecimentos, havendo aqui um efeito sinergístico que suporta a ideia de que as capacidades absortivas são cruciais para explorar efetivamente o know-how externo e obter benefícios das complementariedades entre os recursos internos e externos (HERVAS-OLIVER; ALBORS-GARRIGÓS, 2009). Fica claro, portanto, que a articulação entre esses recursos internos e externos é chave no processo empresarial de criação de valor (MCEVILY; ZAHEER, 1999).

Ademais, a abordagem do cluster utilizada para expressar a concentração geográfica de empresas nesse estudo vai além do que apregoa o mainstream, por entender que além das forças endógenas expressadas por meio das múltiplas interações e fluxos de conhecimentos locais (local buzz), é importante reconhecer o papel dos globalpipelines, que conjuntamente possibilitam a criação de um processo de aprendizagem dinâmico, a produção do conhecimento e processos de inovação que são centrais para o entendimento do sucesso do cluster (BATHELT et al., 2004; HERVAS-OLIVER et al., 2008; HERVAS-OLIVER; BOIXDOMENECH, 2012). Seguindo essa lógica, têm-se que os recursos empresariais e os recursos do cluster são reforçados e complementados pelas interações de conhecimento com agentes externos ao cluster. $\mathrm{O}$ processo de criação do conhecimento torna-se mais valioso na medida em que conhecimentos tácitos e codificados possam ser trocados local e globalmente (BATHELT et al., 2004). Esses pipelines permitem 
conexões entre a empresa e as Redes de Produção Globais (HERVAS-OLIVER; BOIX-DOMENECH, 2012), fator de grande relevância no acesso aos mercados dos países desenvolvidos (GEREFFI et al., 2005).

Enfim, esse estudo demonstra que a criação de valor por parte das empresas geograficamente concentradas deve considerar a dinâmica interna dos recursos e capacidades empresariais; a capacidade de absorção das empresas face aos recursos do cluster (buzz ou atmosfera); por fim, os aspectos que reforçam e complementam a criação de valor proporcionados pelos vínculos externos estabelecidos pelas empresas e atores do cluster.

Acredita-se que a compilação teórica apresentada por meio desse estudo possa estimular o desenvolvimento de trabalhos empíricos que possam reconhecer essa dinâmica e mesmo complementar o entendimento apresentado por esse ensaio, e que esse conteúdo possa auxiliar o desenvolvimento de estratégias que promovam o desenvolvimento nos níveis regional e empresarial. Como sugestões que possam contribuir com o desenvolvimento da área, recomendam-se: estudos descritivos e exploratórios em empresas que pertençam a clusters de diversas naturezas e em diferentes fases do ciclo de vida; estudos que possam mensurar a capacidade absortiva das empresas do cluster e as razões da assimetria na absorção desses recursos; por fim, estudos que demonstrem como os global pipelines reforçam e atualizam as demais fontes de criação de valor do cluster e das empresas.

\section{Referências}

ARIKAN, A. T. Interfirm knowledge exchanges and the knowledge creation capability of clusters. Academy of Management Review, [s.I.], v. 34, n. 4, p. 658-676, Oct. 2009.

ARTUSO, S.B.; LANGRAFE, T.F.; BOAVENTURA, J.M.G. Como surgem clusters? Uma análise da produção internacional sobre o tema. In:SIMPÓSIO DE ADMINISTRAÇÃO DA PRODUÇÃO, LOGÍSTICA E OPERAÇÕES INTERNACIONAIS, 15., 2012. São Paulo. Anais[...]. São Paulo: FAPESP, 2012. p. 1-16.

ASHEIM, B.; GERTLER, M. The Geography of Innovation Systems. In: FAGERBERG, J.; MOWERY, D.C.; NELSON, R. R. The Oxford Handbook of Innovation. Oxford University Press, 2005. p.1-16

BARNEY, J. Firm Resources and Sustained Competitive Advantage. Journal of Management,[s.l.], v. 17, n. 1, p. 99- 120, Special Collection, 1991.

BATHELT, H.; MALMBERG, A.; MASKELL, P. Clusters and Knowledge: Local Buzz, Global Pipelines and the Process of Knowledge Creation. Progress in Human Geography, [s.I.], v. 28, n. 1, p. 31-56, 2004.

BECATTINI, G. The Marshallian district as a socio-economic notion. In: PYKE, F.; BECATTINI, G.; SENGENBERGER, W. (Eds.).Industrial Districts and Inter-firm Co-operation in Italy. Geneva: OMT, 1990. p.13-32.

BRUSCO, S. The Emilian Model: Productive Decentralisation and Social Integration. Cambridge Journal of Economics, [s.I.], v. 6, n. 1, p.167-184, 1982.

DIERICKX, I.; COOL, K. Asset Stock Accumulation and Sustainability of Competitive Advantage. Management Science, [s.I.], v. 35, n.12, p. 1514-1514, 1989.

DUNNING, J.H.; LUNDAN, S.M. Institutions and the OLI paradigm of multinational enterprise.Asia Pacific J Manage, [s.l.], v. 25, n. 1, p. 573-593, 2008.

DYER, J.H.; SINGH, H. The Relational View: cooperative strategy and source of interorganizational competitive advantage. Academy of Management Review, [s.I.], v. 23, n. 4, p. 660-667, 1998.

EISENHARDT, K. M.; MARTIN, J. A. Dynamic capabilities: what are they? Strategic Management Journal, [s.I.], v. 21, n. 1, p. 1105-1121, 2000.

EISINGERICH, A. B.; BELL, S. J.; TRACEY, P. How can clusters sustain performance? The role of network strength, network openness, and environmental uncertainty. Research Policy, [s.I.], v. 39, n. 1, p. 239-253, 2010. 
FENSTERSEIFER, J.E.; WILK, E.O. Visão da Firma baseada em Recursos, Clusters e Performance: um estudo do setor vitivinícola do Rio Grande do Sul. In: ENCONTRO DA ASSOCIAÇÃO NACIONAL DE PROGRAMAS DE PÓS-GRADUAÇÃO EM ADMINISTRAÇÃO, 29., 2005, Brasília. Anais [...]. Brasília: ANPAD, 2005.CD-ROM.

FOSS, N. J. Higher-order industrial capabilities and competitive advantage. Journal of Industry Studies, [s.I.], v. 3, n. 1, p. 1-20, 1996.

GEREFFI, G.; HUMPHREY, J.; STURGEON, T. The Governance of Global Value Chains. Review of International Political Economy, [s.I.], v. 12, n. 1, p. 78-104, Feb. 2005.

GIULIANI, E. The Selective Nature of Knowledge Networks in Clusters: Evidence from the Wine Industry. Journal of Economic Geography, [s.I.], v. 7, n. 1, p. 139-168, 2007.

GIULIANI, E. Network dynamics in regional clusters: evidence from Chile. Research Policy, [s.I.], v. 42, n. 1, p. 1406-1419, 2013.

GIULIANI, E.; BELL, M. The Micro-Determinants of Meso-Level Learning and Innovation: Evidence from a Chilean Wine Cluster. Research Policy, [s.I.], v. 34, n. 1, p. 47-68, 2005.

GRANOVETTER, M. Economic action and social structure: the problem of embeddedness. American Journal of Sociology, [s.I.], v. 91, n. 3, p. 481-510, 1985.

GRANT, R. M. The resource-based theory of competitive advantage: implications for strategy formulation. California Management Review, [s.I.], v. 33, n. 3, p. 114-135, Spring 1991.

HERVAS-OLIVER, J.L.; ALBORS-GARRIGOS, J. Do clusters capabilities matter? Empirical evidence in European clusters. Entrepreneurship and Regional Development, [s.I.], v. 19, n. 2, p. 113-136, 2007.

HERVAS-OLIVER, J.L.; ALBORS-GARRIGOS, J. The role of the firm's internal and relational capabilities in clusters: When distance and embeddedness are not enough to explain innovation. Journal of Economic Geography, [s.I.], v. 9, n. 2, p. 263-283, 2009.

HERVAS-OLIVER, J.L.; ALBORS-GARRIGÓS, J.; DALMAU-PORTA, J. I. External ties and the reduction of knowledge asymmetries among clusters within global value chains: The case of the ceramic tile district of Castellon. European Planning Studies,[s.I.], v. 16, n. 4, p. 507-520, 2008.

HERVAS-OLIVER, J.L. et. al. The role of a firm's absorptive capacity and the technology transfer process in clusters: how effective are technology centers in low-tech clusters? Entrepreneurship and Regional Development, [s.I.], v. 24, n. 7-8, p. 523-559, 2012.

HERVAS-OLIVER, J.L.; BOIX-DOMENECH, R. The Economic Geography of the Meso-global Spaces: Integrating Multinationals and Clusters at the Local-Global Level. European Planning Studies, [s.I.], v. 21, n. 7, p.1-17, 2012.

HUMPHREY, J.; MEMEDOVIC, O. Global Value Chains in Agrifood Sector. Viena: UNIDO, 2006.

IAMMARINO, S.; McCANN, P. The relationship between multinational firms and innovative clusters. In: BOSCHMA, R.; MARTIN, R.The Handbook of Evolutionary Economy Geography. Northampton, MA (USA): Edward Elgar, 2010. CD-ROM

KOGUT, B. The Network as Knowledge: generative rules and the emergence of structure. Strategic Management Journal, [s.I.], v.21, n. 1, p. 405-425, 2000.

KRUGMAN, P. Increasing Returns and Economic Geography. The Journal of Political Economy, [s.I.], v. 99, n.3, p.483-499, 1991.

LASTRES, H. M. M.; CASSIOLATO, J. E.; CAMPOS, R. Arranjos e Sistemas Produtivos e Inovativos Locais: vantagens do enfoque. In: LASTRES, H. M. M.; CASSIOLATO, J. E. Estratégias para o Desenvolvimento: um enfoque sobre Arranjos Produtivos Locais do Norte, Nordeste e Centro-Oeste Brasileiros. Rio de Janeiro: E-papers, 2006. CO-ROM 
LAZZERETTI, L; SEDITA, S.R.; CALOFFI, A. Founders and Disseminators of Cluster. Research. Journal of Economic Geography, [s.I.], v. 14, n. 1, p. 1-23, 2013.

MALMBERG, A.; MASKELL, P. The Elusive Concept of Localization Economies - Towards a Knowledgebased Theory of Spacial Clustering.Environment and Planning. [s.I.], v. 34, n. 3, p. 429-449, 2002.

MALMBERG, A.; MASKELL, P. Localized Learning Revisited.Growth and Change, [s.I.], v. 37, n.1, p.118, 2006.

MALMBERG, A.; MASKELL, P. An Evolutionary Approach to Localized Learning and Spatial Clustering. In: BOSCHMA, R.; MARTIN, R. The Handbook of Evolutionary Economy Geography. Northampton, MA (USA): Edward Elgar, 2010. CD-ROM

MARSHALL A. Principles of Economics. 8th Ed. Macmillan; London, 1920.

MASKELL, P.; MALMBERG, A. Localized learning and industrial competitiveness, Cambridge Journal of Economics, [s.I.], v. 23, n. 1, p. 167-185, 1999.

MCCANN, B.; FOLTA, T. Location matters: where we have been and where we might go in agglomeration research. Journal of Management, [s.I.], v. 34, n. 1, p. 532-565, 2008.

MCEVILY, B.; ZAHEER, A. Bridging ties: a source of firms heterogeneity in competitive capabilities. Strategic Management Journal,[s.I.], v. 20, n.1, p.1133-1156, 1999.

MOLINA-MORALES, X.; MARTINEZ-FERNANDEZ, T. How much difference is there between industrial district firms? A net value creation approach. Research Policy, [s.I.], v. 33, n.1, p. 473-486, 2004.

NELSON, R. R. The Evolution of Competitive or Comparative Advantage: A Preliminary Report on a Study. Industrial and Corporate Change, [s.I.], v. 5, n.2, p.597-617, Feb. 1996.

NELSON, R. R. Bringing institutions into evolucionary growth theory. Journal of Evolucionary Economics, [s.l.], v. 12, n. 1, p.17-28, 2002.

NELSON, R. R.; SAMPAT, B.N. Making sense of institutions as a factor shaping economic performance. Journal of Economic Behavior \& Organization, [s.I.], v. 44, n.1, p.31-54, 2001.

NELSON, R.R.; WINTER, S.G.An evolutionary theory of economic change. Cambridge: Harvard University, 1982.

NORTH, D. Institutions, Institutional Change and Economic Performance. Cambridge: Cambridge University Press, 1990.

PENG, M.W.; WANG, D.Y.L.; JIANG, Y. An Institution-based View of International business strategy: a focus on emerging economies. Journal of International Business Studies, [s.I.], v. 39, n. 1, p. 920-936, 2008.

PENROSE, E. The theory of growth of the firm. New York: Oxford University Press, 1995.

PETERAF, M. A. The Cornestones of Competitive Advantage: a Resource-Based View. Strategic Management Journal, [s.I.], v. 14, n. 1, p. 179-191, 1993.

PETERAF, M.; STEFANO, G.; VERONA, G. The Elephant in the Room of Dynamic Capabilities: Bringing two Diverging Conversations Together. Strategic Management Journal, [s.I.], v. 34, n. 12, p. 1389-1410, 2013.

PORTER, M. E. Clusters and the New Economics of Competition. Harvard Business Review, [s.I.], v. 76, n. 6,, p. 79-91, Nov./Dec. 1998.

PORTER, M. E.Competitive Advantage: creating and sustaining superior performance. New York: The Free Press, 1985.

PORTER, M. E. Location, Competition, and Economic Development: Local Clusters in a Global Economy. Economic Development Quarterly, [s.I.], v.14, n. 1, February, p. 15-34, 2000. 
PORTER, M. E. The Contributions of Industrial Organization to Strategic Management: Academy of Management.The Academy of Management Review, [s.I.], v. 6, n. 4, p. 609-620, Oct. 1981.

PORTER, M. E. The Structure Within Industries and Companies' Performance. Review of Economics Land Statistics, [s.I.], v. 61, n. 2, p. 214-227, May 1979.

PORTER, M. E. Towards a Dynamic Theory of Strategy. Strategic Management Journal, [s.I.], v. 12, n.1, p. 95-117, 1991.

RIGBY, D. L.; BROWN, W. M. Who Benefits from Agglomeration? Regional Studies, [s.I.], v. 49, n. 1, p. 28-43, 2013.

SFORZI, F. Unas realidades ignoradas: de Marshall a Becattini. Espanha: Mediterraneo Económico, 2008.

SUZIGAN, W.; GARCIA, R.; FURTADO, J. Sistemas locais de produção/inovação: metodologia para identificação, estudos de casos e sugestões de políticas. In: DINIZ, C.C.; LEMOS, M.B. Economia e Território. Belo Horizonte: Ed. UFMG, 2005. p. 131-170

TALLMAN, S. et. al. . Knowledge, Clusters, and Competitive Advantage. The Academy of Management Review, [s.l.], v. 29, n. 2, p. 258-271, 2004.

TEECE, D. J.; PISANO, G.; SHUEN, A. Dynamic capabilities and strategic management. Strategic Management Journal, [s.I.], v. 18, n. 7, p. 509-533, 1997.

TER WAL, A. L.J. Cluster Emergence and Network Evolution: a Longitudinal Analysis of the Inventor Network in Sophia-Antipolis. Regional Studies, [s.I.], v. 47, n. 5, p.651-668, 2013.

TER WAL, A. L.J.; BOSCHMA, R. Co-evolution of Firms, Industries and Networks in Space. Regional Studies, [s.I.], v. 45, n. 7, p. 919-933, 2011.

WERNERFELT, B. A resource-based view of the firm. Strategic Management Journal, [s.I.], v. 5, n. 1, p. 171-180, 1984.

ZEN, A. C. A Influência dos Recursos na Internacionalização de Empresas inseridas em Clusters: uma pesquisa no setor vitivinícola no Brasil e na França. 2010. Tese (Doutorado em Administração) Universidade Federal do Rio Grande do Sul, Escola de Administração, Porto Alegre, 2010.

Submetido em: 20/04/2018

Aceito em: 01/02/2019 